\title{
Self-Treated Hypoglycemia in Type 2 Diabetes Mellitus: Results from the Second Wave of an International Cross-Sectional Survey
}

\author{
Meryl Brod • Gagik Galstyan • Ambika Gopalakrishnan Unnikrishnan • \\ Ilana Harman-Boehm • Vinay Prusty · Fernando Lavalle • \\ Margaret McGill · Angela Murphy · Felix Puchulu
}

Received: January 7, 2016 / Published online: March 21, 2016

(C) The Author(s) 2016. This article is published with open access at Springerlink.com

\begin{abstract}
Introduction: The aim of this study was to assess the total frequency of self-treated hypoglycemia in type 2 diabetes mellitus patients using regimens including basal insulin analogs, and to describe the psychological impact and behavioral response to these events from the perspective of patients and
\end{abstract}

Enhanced content To view enhanced content for this article go to http://www.medengine.com/Redeem/6884 F0600B63DE7F.

M. Brod ( $\square)$

The Brod Group, Mill Valley, CA, USA

e-mail: mbrod@thebrodgroup.net

G. Galstyan

Endocrinological Research Center, Moscow,

Russian Federation

A. G. Unnikrishnan

Chellaram Diabetes Institute, Pune, Maharashtra, India

I. Harman-Boehm

Department of Internal Medicine and the Diabetes

Unit, Soroka University Medical Center, Beer-Sheva, Israel

V. Prusty

Novo Nordisk Pharma Korea, Seoul, Korea prescribers (i.e., hospital specialists and primary care physicians).

Methods: The global attitude of patients and physicians 2 (GAPP2) survey was an online multinational, cross-sectional survey of patients with type 2 diabetes mellitus treated with basal insulin analogs, with or without bolus insulin. Prescribers directly involved in the care of these patients were also surveyed. Here, we report the results of the second wave of the GAPP2 survey, in which the primary variable of interest was self-treated hypoglycemia.

Results: A total of 855 patients and 1003 prescribers, from 7 countries, completed the

F. Lavalle

Autonomous University of Nuevo León, Pedro de Alba S/N, Ciudad Universitaria, San Nicolás de los Garza, Nuevo León, Mexico

M. McGill

Sydney Nursing School, University of Sydney, Sydney, New South Wales, Australia
A. Murphy
Sunward Park Medical Centre, Johannesburg,
South Africa
F. Puchulu
Hospital de Clínicas, University of Buenos Aires, Buenos Aires, Argentina 
survey. Overall, $28 \%$ of patients had experienced self-treated hypoglycemia during the previous 30 days, with two-thirds of events occurring during the day and one-third of events occurring nocturnally. Prescribers reported discussing events with 55\% of patients over this period. Patients worried about self-treated hypoglycemia in a range of situations, and prescribers under-estimated this worry. Many patients who had experienced self-treated hypoglycemia in the last 30 days reported missing (19\%), mistiming (7\%), or reducing $(7 \%)$ their basal insulin dose as a result.

Conclusion: Self-treated hypoglycemia was relatively common in patients using basal insulin analogs, with or without bolus insulin. Whilst the frequency of hypoglycemia was greater during the daytime than at night, patients worried more about nocturnal events and this level of worry was under-estimated by physicians. Additional advice and support may be needed for both patients and prescribers, to reduce the frequency and impact of self-treated hypoglycemia.

Funding: Novo Nordisk.

Keywords: GAPP2; Type 2 diabetes mellitus; Self-treated hypoglycemia; Minor hypoglycemia; Nocturnal hypoglycemia

\section{INTRODUCTION}

The use of insulin to reduce blood glucose levels in type 2 diabetes mellitus (T2DM) is highly effective, but can be challenging (e.g., taking time to develop an appropriate regimen) and the risk of hypoglycemia remains an important consideration for both physicians and patients [1-3].

Severe hypoglycemia, defined as an event requiring the assistance of another person to actively administer carbohydrates, glucagon, or take other corrective actions, is a key cause of morbidity in T2DM and an important barrier to optimal glycemic control [4]. Less severe hypoglycemic events that the individual can self-treat by consuming fast-acting carbohydrate, known as 'self-treated' or 'minor' hypoglycemic events, are not always recognized by the individual or their clinical team as being clinically significant. Data suggest that self-treated hypoglycemic events, whether they occur in the daytime or at night, have an impact on patient functioning, well-being and diabetes management [5-7]. Of particular importance is the relationship between, often unnoticed, nocturnal hypoglycemia and poor health outcomes including adverse cardiovascular events [8]. Self-treated hypoglycemia also has substantial economic consequences for patients and their employers due to lost working hours and reduced productivity [7]. Recently, reported data from Denmark suggest that $9 \%$ of self-treated episodes lead to lost work time [9]. In the UK, self-treated hypoglycemia is estimated to cost the National Health Service $£ 172.1$ million per year [10]. Recent research in the USA found that non-severe episodes incur a cost of $\$ 11$ per episode [11].

Research has also suggested that around one-third of patients are very worried about hypoglycemia, and a similar proportion maintain their blood glucose levels within a 'safety margin' at levels above recommended targets, in an attempt to reduce the risk of hypoglycemia [8]. Given the important effect of these events on both patient functioning and diabetes management, the prevalence and impact of self-treated hypoglycemic events among patients with T2DM requires additional study. For this reason, the Global Attitude of Patients and Physicians 2 (GAPP2) web-based 
survey was initiated. The first wave of the online survey was initially conducted among patients treated with basal or basal plus bolus insulin analogs and relevant prescribers (i.e., hospital specialists and primary care physicians) in six countries: Canada, Denmark, Germany, Japan, UK, and USA. One of the key aims of the first wave was to estimate the prevalence of self-treated hypoglycemia in patients and identify demographic, treatment-related and behavioral risk factors associated with these events. The key findings from the first wave of the survey have been published elsewhere $[3,6$, 12, 13].

In wave two, reported here, the survey was extended to further countries in Europe, North and South America, Asia, Africa, and Australia. Similar to the first wave, the primary aim was to collect and assess data regarding self-treated hypoglycemia in users of basal insulin analogs, with or without bolus insulin, from both the patient and prescriber's perspective.

\section{METHODS}

\section{Recruitment}

All procedures conducted were in accordance with the ethical standards of the responsible committee on human experimentation (institutional and national) and with the Helsinki Declaration of 1964, as revised in 2013. Informed consent was obtained from all patients included in the survey. Data were collected between September 2012 and January 2013. In total, 11 countries were included in the second wave of the survey. This manuscript focuses on pooled data from seven countries: Argentina, Australia, India, Israel, Mexico, Russia, and South Africa. Data from these countries were pooled as they belonged to the region of international operations of the study sponsor.
Patient participants were recruited from online general population research panels, using recruitment techniques representative of the local online community including banner advertisements, e-mail campaigns, blogs, social media, TV/print and SMS campaigns. Patient eligibility criteria required being diagnosed with T2DM over the age of 40 years and being on a long-acting basal insulin analog alone, or on long-acting basal and short-acting bolus insulin analogs taken separately. To maintain consistency and enable comparison with the results from the first wave of the GAPP2 survey, patients on bolus only, premix insulin or using insulin pumps were excluded $[3,6,12,13]$.

Prescribers were recruited from pre-existing online healthcare professional research panels and were initially targeted by specialty (i.e., primary care, specialist, or other). They were then screened to ensure that they treated a minimum number of patients with diabetes in a typical month. Criteria varied by country, but typically 20 patients per month for general practitioners (GPs) and 40 patients per month for hospital specialists were sufficient for inclusion.

\section{Survey}

The survey materials have previously been described in greater detail in the first wave of the GAPP2 survey $[3,6,12,13]$. Survey items were generated from multiple data sources: an international steering committee of diabetes clinical experts, relevant current literature, and from key concepts and themes from the transcripts of nine previously conducted focus groups and interviews with patients with diabetes. Questionnaires for both patients (90 items) and physicians (58 items) were structured in the same way to facilitate between-group comparisons. Items covered 
demographics and background information, diabetes management, self-treated hypoglycemia and patient functioning and well-being. Self-treated hypoglycemic events (i.e., 'hypos') were defined as symptoms of low blood sugar (i.e., sweating, weakness, trembling or difficulty concentrating) that patients could treat by themselves by drinking a glass of juice, eating, or taking a sugar pill. Patients were specifically asked about the characteristics, incidence and impact of such events, with physicians being asked to answer all questions by thinking about their own patients who were prescribed basal insulin analogs, with or without bolus insulin.

To minimize any recall bias on patient responses, participants were asked to only report events that occurred during the 30 days prior to completion of the survey. Participants were offered an "I don't know" answer where applicable to avoid forcing inaccurate responses, and all data were logic-tested to ensure that participants did not provide contradictory answers. Data identified electronically as being incomplete were collected but not processed or included in the analyses.

\section{Statistical Analysis}

Statistical analysis was conducted using statistical analysis software SAS (SAS Institute Inc., Cary, NC, USA). To describe the patient and prescriber population, the data were descriptively analyzed using frequencies and mean values. Paired $t$ tests were used to show the actual differences between groups where applicable. Pearson's Chi squared test was used for non-parametric data. Outliers were identified by taking a range from either the mean or median score, depending on the question type. Where notable numbers of outliers were seen to be affecting the results for a given question, the question was assessed subjectively and responses outside the statistically acceptable range deemed not plausible in the context of the question were removed.

\section{RESULTS}

\section{Participants}

A total of 509,692 participants were invited to participate in the survey and 2271 prescribers and 15,180 patients responded. Of these, 855 patients and 1003 prescribers were eligible for and completed the survey (Fig. 1). The mean age of the patient sample was 57.1 years, with a mean diabetes duration of 8.2 years. The prescriber sample treated a mean of 127.7 patients aged $>40$ years per month. Table 1 summarizes the full characteristics of the sample.

\section{Total Frequency of Self-Treated Hypoglycemia}

For the sample as a whole, $28 \%$ of respondents reported that their last event was during the last 30 days and the mean number of events per patient was 3.8 per month. For daytime self-treated hypos, the mean number of events per patient was 2.6 per month. For nocturnal self-treated hypos, the mean number of events per patient was 1.3 per month (Table 2).

\section{Discussion of Self-Treated Hypoglycemia}

Totals of $59 \%$ and $42 \%$ of all respondents reported that self-treated hypos were typically discussed during consultations with specialists and GPs, respectively. Prescribers reported that they had only discussed or reviewed minor 


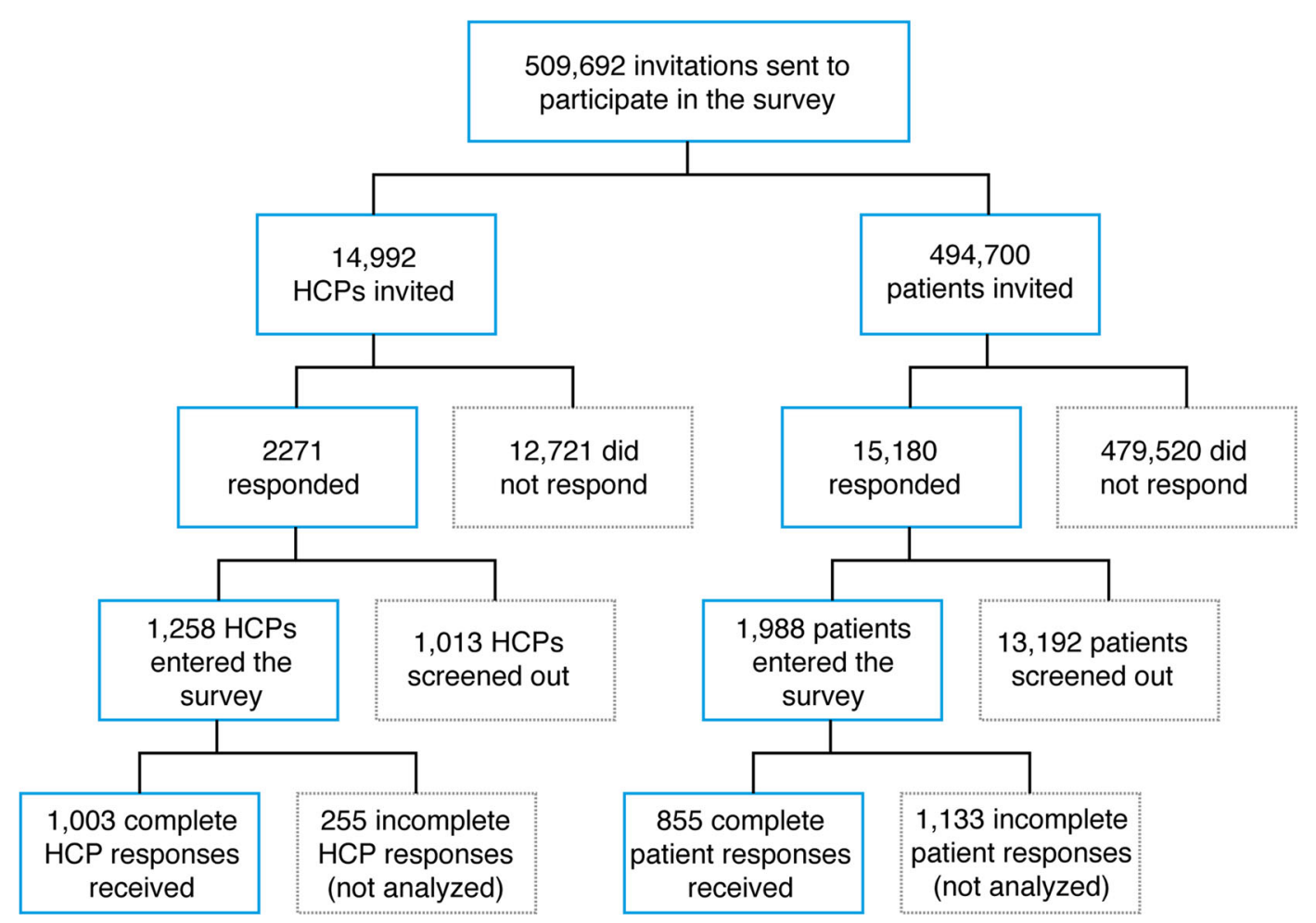

Fig. 1 Patient and prescriber survey recruitment flow diagram. HCP healthcare professional

hypos with $55 \%$ of the patients that they had seen in the past 30 days. When prescribers were asked to describe how often they discussed minor hypos with these patients, only $57 \%$ said they 'always' or 'most of the time' discussed with patients on basal only insulin and 69\% said they 'always' or 'most of the time' discussed with patients on basal-bolus insulin (Fig. 2).

Prescribers were asked why they chose not to discuss minor hypos with some of these patients. With basal only patients, the most commonly cited reasons were: patients are already well educated about minor hypos (72\%); patients do not report minor hypos (44\%); and a lack of time during consultation (29\%). When asked about basal-bolus patients, the same three responses were reported $(67 \%$, $34 \%$, and $31 \%$, respectively). Prescribers also estimated that about one-quarter of their patients under-reported the frequency (31\%) or severity $(24 \%)$ of minor hypos they had experienced.

\section{Impact of Self-Treated Hypoglycemia}

Patients were asked how long it took to perceive that they had regained normal physical and mental functioning after a self-treated hypo. The time to recover physical and mental functioning was believed by $32 \%$ and $45 \%$ of patients, respectively, to be longer than $1 \mathrm{~h}$. For prescribers, 25\% reported that they believed it took their patients longer than $1 \mathrm{~h}$ to recover normal physical functioning and $43 \%$ reported they believed it took their patients longer than $1 \mathrm{~h}$ to regain mental functioning. Areas of functioning that patients felt were particularly affected by self-treated hypos were 'performance at work', 'ability to focus and concentrate,' and 'taking part in sport and 


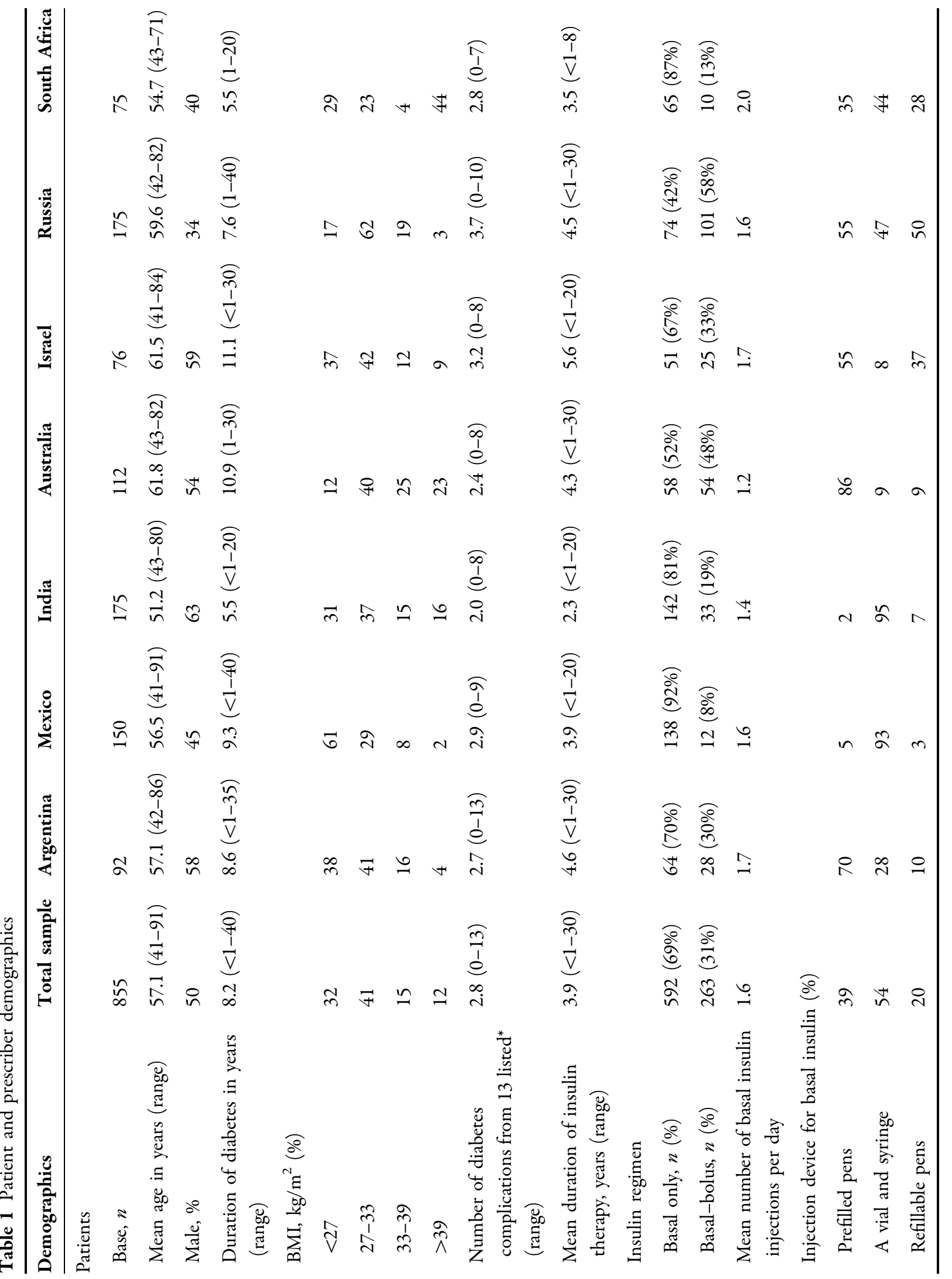




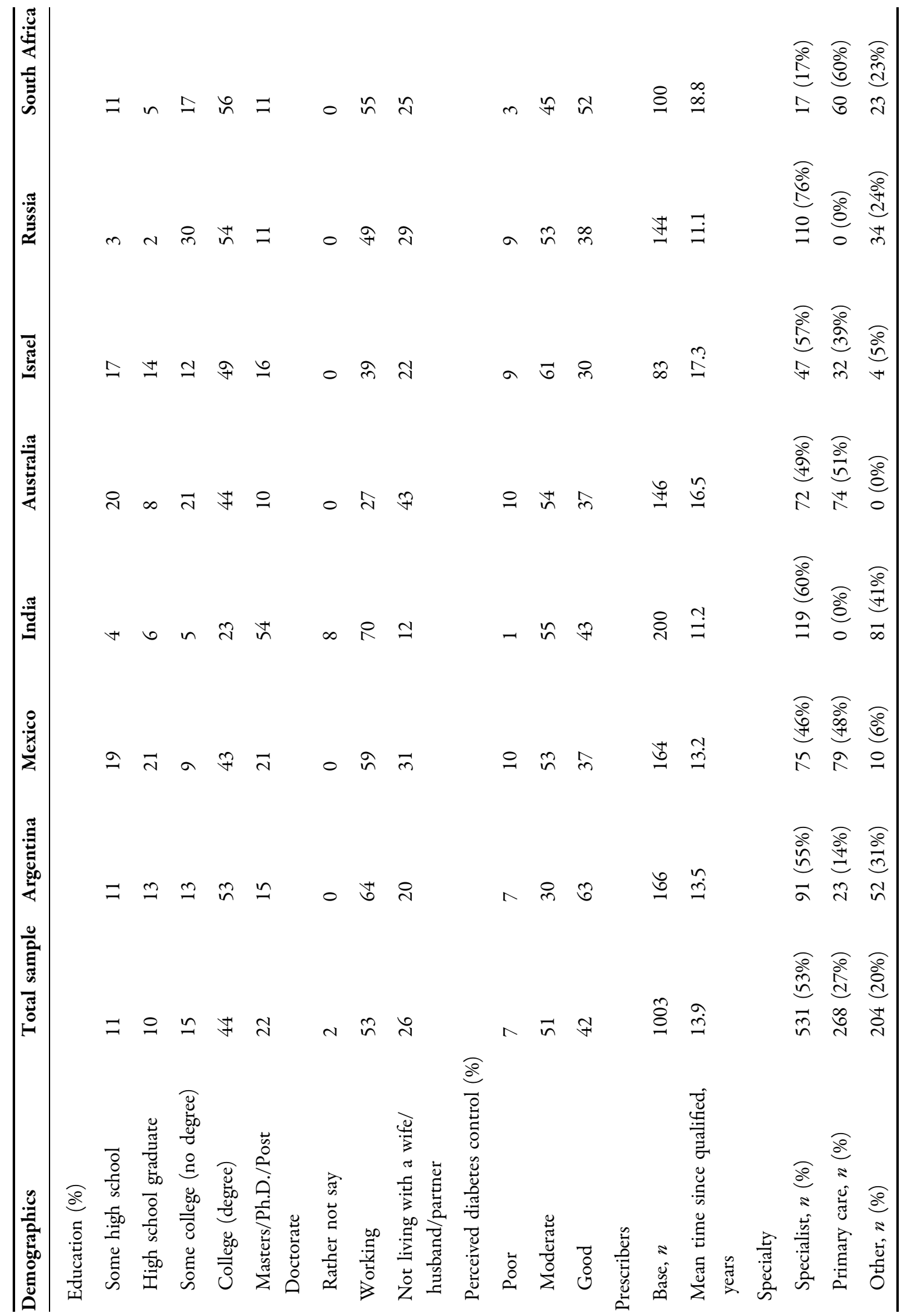




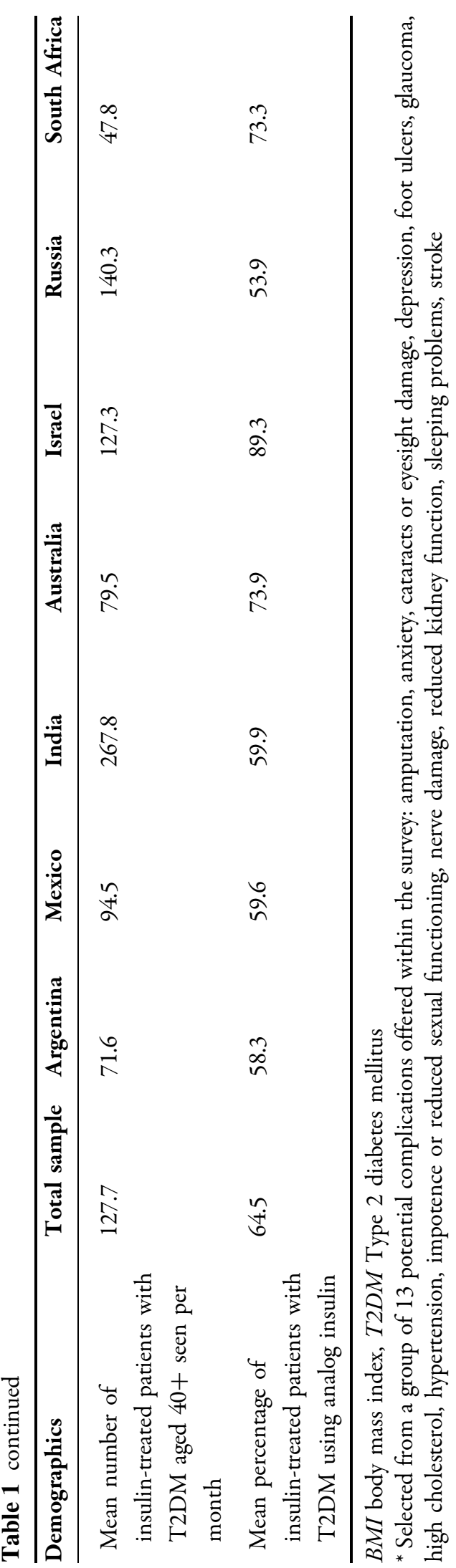

exercise' $(47 \%, 45 \%$, and $45 \%$ of patients, respectively, reporting a 'significant' or 'somewhat' negative impact).

\section{Levels of Worry About Self-Treated Hypoglycemia}

Patients worried about self-treated hypos in a wide variety of circumstances: more than two-thirds of patients admitted to being 'very' or 'somewhat' worried about events during each of the following daily situations: 'somewhere where there is no easy access to food or drink' (75\% of patients), 'when driving' (70\%), 'while sleeping' (68\%), 'when alone at home' (68\%), and 'while caring for children/grandchildren' (68\%). Patients also stated that they were more likely to worry about nocturnal self-treated hypos than daytime events: $68 \%$ versus $57 \%$, respectively, reported they were 'very' or 'somewhat worried'.

Prescribers appeared to underestimate this worry; for each of the five daily scenarios described above, fewer than $30 \%$ of prescribers felt that their patients worried about minor hypos: 'while sleeping' (29\%), 'when alone at home' (19\%), 'when driving' (19\%), 'somewhere where there is no easy access to food or drink' (18\%), and 'while caring for children/grandchildren' (12\%).

When prescribers were asked to state the situation in which they felt their patients worried the most about minor hypos, the most common response was 'whilst sleeping' (Fig. 3). However, half of prescribers reported that they believed that less than $25 \%$ of their patients worried about nocturnal minor hypos.

\section{Response to Self-Treated Hypoglycemia}

A substantial proportion of patients reported adjusting their own treatment regimen in 
Table 2 Frequency of self-treated hypoglycemia among patients in the last 30 days

\begin{tabular}{|c|c|c|c|c|c|c|c|c|}
\hline & $\begin{array}{l}\text { Total } \\
\text { sample }\end{array}$ & Argentina & Mexico & India & Australia & Israel & Russia & $\begin{array}{l}\text { South } \\
\text { Africa }\end{array}$ \\
\hline Effective base $(n)$ & 776 & 86 & 143 & 145 & 108 & 68 & 163 & 63 \\
\hline $\begin{array}{l}\% \text { of patients with at } \\
\text { least one event }(n)\end{array}$ & $28 \%(217)$ & $31 \%(27)$ & $47 \%(67)$ & $14 \%(21)$ & $33 \%(36)$ & $29 \%(20)$ & $25 \%(40)$ & $10 \%(6)$ \\
\hline \multicolumn{9}{|l|}{$\begin{array}{l}\text { All self-treated } \\
\text { hypoglycemia* }\end{array}$} \\
\hline $\begin{array}{l}\text { Mean number of events } \\
\text { per patient (range) }\end{array}$ & $3.8(1-25)$ & $5.0(1-15)$ & $3.3(1-10)$ & $4.5(1-12)$ & $3.2(1-20$ & $3.1(1-20)$ & $4.1(1-25)$ & $6.8(1-23)$ \\
\hline $\begin{array}{l}\% \text { of patients with } \\
5+\text { events }(n)\end{array}$ & $25 \%(55)$ & $52 \%(14)$ & $22 \%(15)$ & $29 \%(6)$ & $17 \%(6)$ & $20 \%(4)$ & $18 \%(7)$ & $50 \%(3)$ \\
\hline \multicolumn{9}{|l|}{$\begin{array}{l}\text { Daytime self-treated } \\
\text { hypoglycemia* }\end{array}$} \\
\hline $\begin{array}{l}\text { Mean number of events } \\
\text { per patient (range) }\end{array}$ & $2.6(0-25)$ & $2.9(0-10)$ & $2.0(0-8)$ & $2.5(0-6)$ & $2.8(0-20)$ & $2.0(0-10)$ & $3.0(0-25)$ & $5.5(1-20)$ \\
\hline $\begin{array}{l}\% \text { of patients with } \\
5+\text { events }(n)\end{array}$ & $13 \%(29)$ & $26 \%(7)$ & $9 \%(6)$ & $5 \%(1)$ & $14 \%(5)$ & $20 \%(4)$ & $13 \%(5)$ & $17 \%(1)$ \\
\hline \multicolumn{9}{|l|}{$\begin{array}{l}\text { Nocturnal self-treated } \\
\text { hypoglycemia* }\end{array}$} \\
\hline $\begin{array}{l}\text { Mean number of events } \\
\text { per patient (range) }\end{array}$ & $1.3(0-10)$ & $2.1(0-7)$ & $1.3(0-5)$ & $2.0(1-8)$ & $0.4(0-3)$ & $1.1(0-10)$ & $1.1(0-6)$ & $1.3(0-3)$ \\
\hline $\begin{array}{l}\% \text { of patients with } \\
5+\text { events }(n)\end{array}$ & $6 \%(12)$ & $19 \%(5)$ & $3 \%(2)$ & $14 \%(3)$ & $0 \%(0)$ & $5 \%(1)$ & $3 \%(1)$ & $0 \%(0)$ \\
\hline
\end{tabular}

* Among patients who had experienced at least one episode of self-treated hypoglycemia (daytime or nocturnal) in the last 30 days

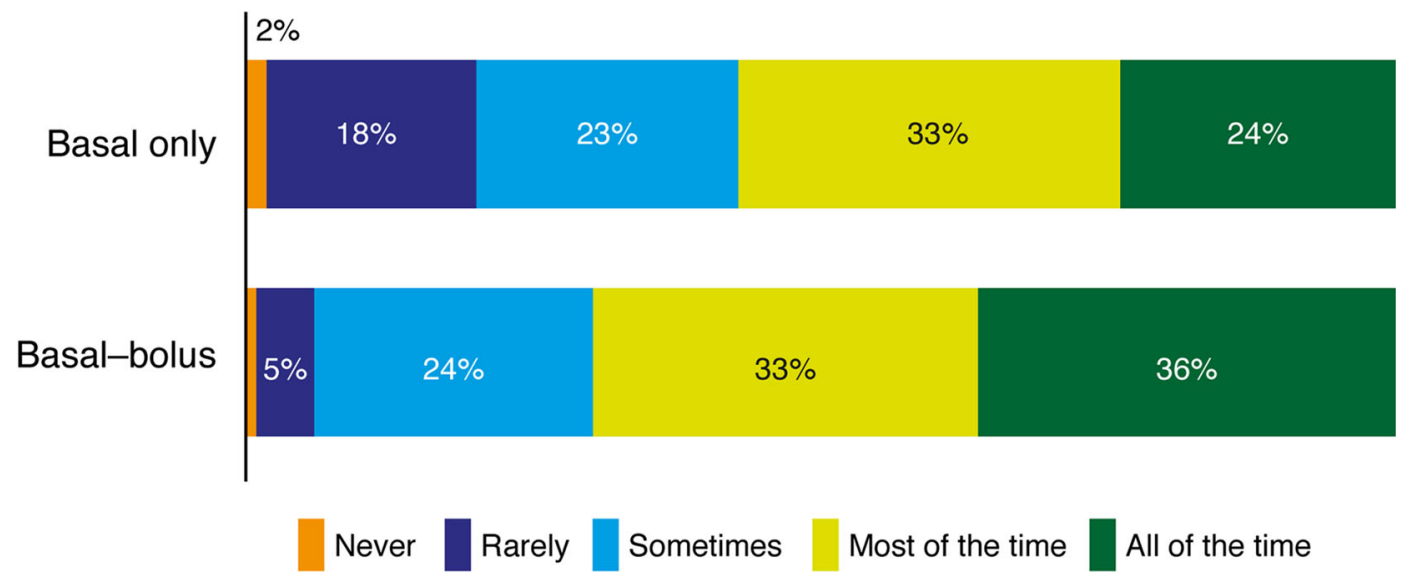

Fig. 2 Frequency with which prescribers discuss minor hypoglycemia with their patients

response to self-treated hypos. Among patients who had ever experienced such an event, 19\%, $15 \%$, and $13 \%$, respectively, reported that they responded by missing, mistiming ( $>2 \mathrm{~h}$ earlier or later than prescribed), or reducing a dose of their basal insulin. Among patients who had experienced such an event in the past 30 days, 19\% (range 1-30 occasions), 7\% (range 1-30 


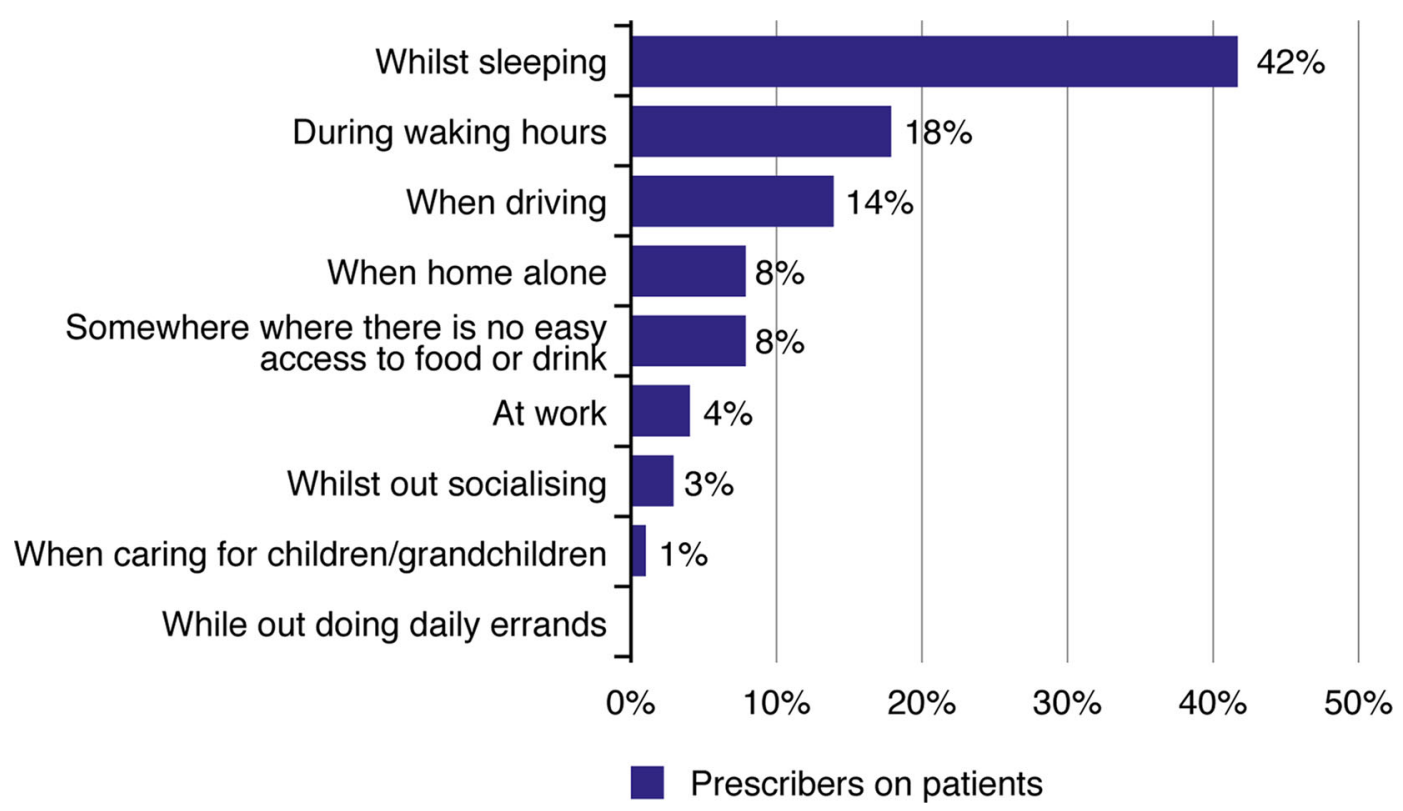

Fig. 3 Situations in which prescribers feel their patients with type 2 diabetes on insulin analogs worry most about self-treated hypoglycemia

occasions), and 7\% (range 1-30 occasions), respectively, reported missing, mistiming, or reducing a dose of their basal insulin in response. On average, around $25 \%$ of patients reported increasing the frequency of blood glucose monitoring in response to self-treated hypos (Fig. 4).

A fear of nocturnal hypos appeared to be a particularly common reason for insulin misdosing. A total of $22 \%$ of patients reported that they had intentionally let their blood sugar level go higher than it should to reduce the risk of nocturnal self-treated hypos. A similar proportion $(22 \%)$ reported that they had, at some time, not taken their insulin exactly as prescribed due to fear of nocturnal hypos.

Prescribers were asked to describe the recommendations they gave to patients who experienced a number of minor hypos. The actions that most physicians reported they undertook 'most or all of the time' were educational: 'educate the patient on how to avoid hypoglycemia' (80\% of prescribers), 'educate the patient on how to recognize hypoglycemia' (79\%), and 'advise them on how to self-manage hypoglycemia' (77\%). Prescribers reported they were likely to adjust the treatment regimen 'most or all of the time' in such cases: 'reduce the dose of basal insulin temporarily until they have restored hypoglycemia awareness' (23\%), 'reduce the dose of basal insulin long-term' (14\%), 'split the basal insulin into two doses' (4\%), 'switch the type of insulin they are on' (3\%).

Self-treated hypos were also found to have an impact on healthcare resources; many patients who had experienced a self-treated hypo reported making unplanned trips to a diabetes specialist (26\%), a primary healthcare provider (25\%), or a hospital emergency department (14\%). Further, a majority of prescribers $(58 \%)$ reported that they were contacted at least once per month as a result of a patient experiencing a minor hypo, while $15 \%$ reported that they were contacted at least once per week. 


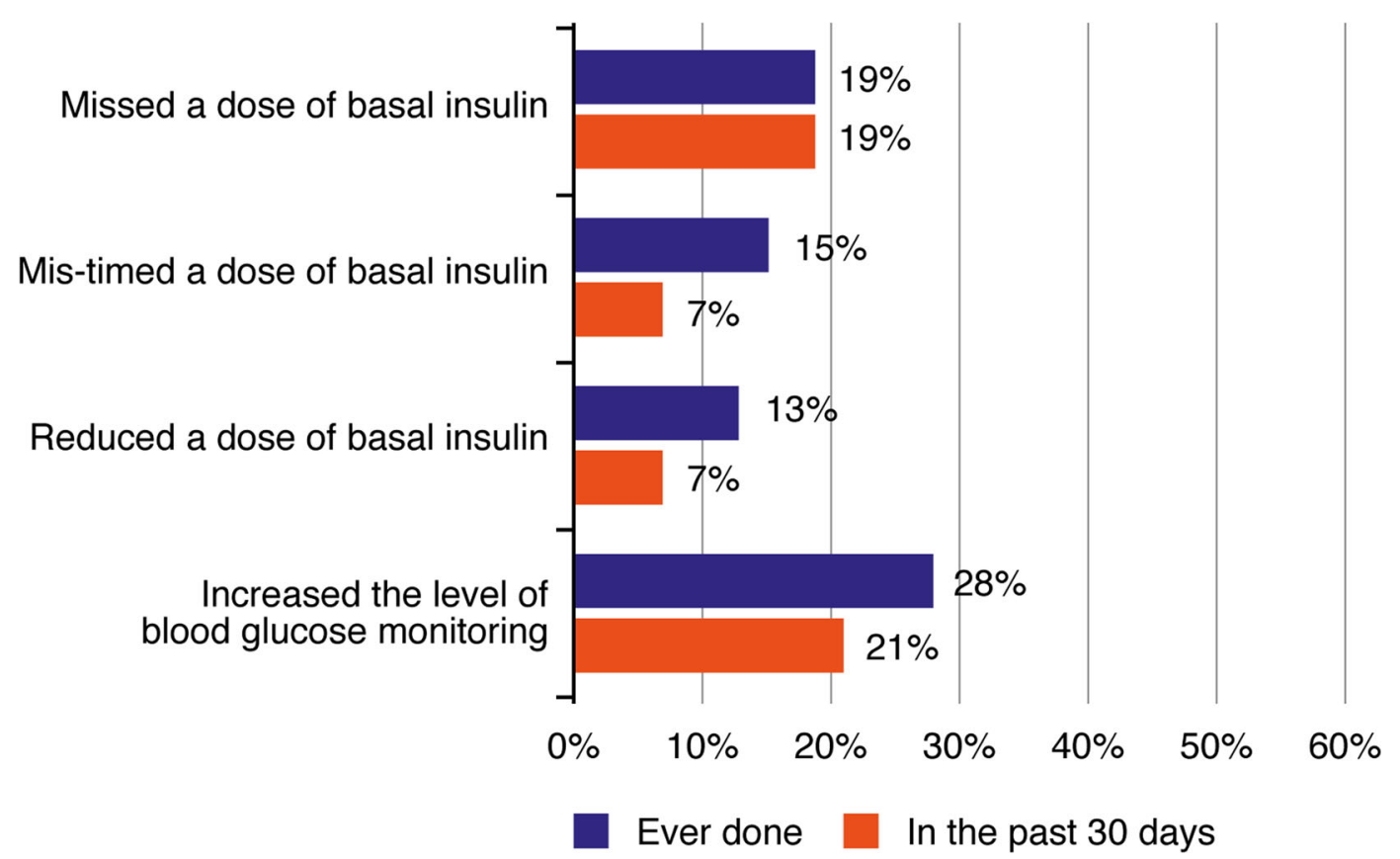

Fig. 4 Frequency of specific responses to self-treated hypoglycemia (ever experienced and last 30 days) among patients on basal insulin analogs

\section{DISCUSSION}

This manuscript reports data from the second wave of the GAPP2 survey, which specifically examined the cumulative prevalence and impact of self-treated hypos among patients with T2DM treated with basal insulin analogs, with or without bolus insulin. The results corroborate many of the findings from the first wave of the GAPP2 survey [3, 6, 12, 13]. With regard to the incidence of self-treated hypos (i.e., missing, mistiming, or reducing doses), $28 \%$ of patients reported that they had experienced an event in the last 30 days. While this is lower than the $36 \%$ of patients who reported events in the first wave, it confirms that self-treated hypos are still relatively common in the lives of those with T2DM who are receiving treatment with insulin analogs.

Similar patterns of behavior in response to self-treated hypos were reported in the current survey and in the first wave, but a notably higher incidence of missed doses was observed in the current survey compared with the first wave (i.e., 19\% versus 7\%, respectively). The causes of this higher incidence observed in the second wave countries require further research. As in the first wave, we observed a higher rate of worry regarding nocturnal hypos compared with daytime events [3]. It is of note that the seven countries represented in the current survey differ substantially from those providing data in the first wave in terms of their culture, health systems and economics. It is therefore of interest that the psychological and behavioral findings from the current survey align with those of the more homogenous first wave cohort. This suggests that responses to self-treated hypos are not culture-specific and can be generalized worldwide.

Research has suggested that as few as 10 symptomatic, non-severe hypos per year can have a clinically relevant impact on functioning 
(e.g., work productivity). Further, the psychological impact of these events increases with their frequency $[14,15]$. Extrapolation of the mean number of minor self-treated hypos observed in the current survey (i.e., 3.8 events over 30 days) suggests that many patients could be experiencing more than 40 self-treated hypos per year. Moreover, almost half of patients and physicians felt that the time taken to recover normal mental functioning after a minor hypo was greater than one hour, highlighting the non-trivial impact that these events can have on daily functioning. Hence, these results would indicate there is a need for enhanced surveillance and greater patient education when consulting with individuals at higher risk for minor hypos.

The results also indicate that prescribers greatly under-estimated levels of concern among their patients with regard to self-treated minor hypos. For example, while $75 \%$ of patients reported that they worried about self-treated hypos in situations where there was no easy access to food or drink, only $18 \%$ of physicians felt that patients worried about this scenario. This discrepancy suggests that prescribers may need to adopt a more proactive approach to addressing and managing their patients' concerns regarding hypoglycemia, particularly with patients who have recently experienced a minor hypo, as these individuals often experience a greater fear of future events [16, 17].

Further, the impact of these events on general diabetes management must be considered. For some patients it may be necessary to adjust clinical management to account for their behavioral and psychological responses to events, since these responses include reducing or missing insulin doses and deliberately allowing blood glucose levels to rise. It is noteworthy that more than three-quarters of prescribers reported that they would usually respond to a patient experiencing repeated minor hypos by educating them on recognizing/avoiding and self-managing such events. However, few prescribers reported they would typically adjust the treatment regimen (e.g., switching the type of insulin or reducing the basal insulin dose temporarily or long-term). Such adjustments may be clinically appropriate in some cases, and it is important that prescribers remain vigilant to the potential threat of minor hypos. In this survey, prescribers reported that they had discussed minor hypos with only half of the patients they had seen in the past 30 days. It is thus likely that a greater focus on discussing minor hypos at initiation and/or early in insulin treatment could help to reduce the frequency of these events.

The survey had a number of important limitations that should be discussed. To enable comparison with the first wave of the GAPP2 survey $[3,6,12,13]$, we employed the same web-based methodology and this could be argued to have produced a selection bias. Namely, only literate participants with internet access were able to participate and it is likely that internet accessibility varied between the different countries that were sampled. This may have resulted in local over-representations of specific demographic groups with internet access, such as younger patients and those in office-based employment-particularly in developing countries where internet access is more limited. As an example, the Indian sample was more than 10 years younger and more than twice as likely to be employed when compared with the Australian sample. Moreover, internet access may still be an indicator of socioeconomic status and access to quality medical care, including the availability of 
prescription medication. Overall, these factors would suggest that the survey participants were not fully representative of the insulin-treated diabetes population in their respective countries.

Consequently, future research is now needed to survey more representative cohorts through the use of alternative methods (e.g., face-to-face interviews) and the inclusion of different insulin regimens (e.g., insulin pump therapy and premix insulin). For instance, in India, premix insulin is the preferred insulin treatment for $\mathrm{T} 2 \mathrm{DM}$, and the frequency and impact of self-treated hypos within this patient group is of clinical interest. As this survey was not designed to examine country-specific differences, further research is also needed to better understand the potential influence of culture and healthcare systems on the frequency of minor events, along with their psychological and behavioral outcomes. We also did not include a baseline measure of $\mathrm{HbA}_{1 \mathrm{c}}$, which would have allowed for the relationship between level of glycemic control, frequency and impact of self-treated hypos to be examined.

In addition, the survey was self-reported, which may have led to systematic errors (e.g., recall bias) that affected the accuracy of the responses. Attempts were made to mitigate this by focusing on self-treated minor hypos from the past 30 days only, and previous surveys and findings from focus groups conducted prior to GAPP2 have concluded that the recall of hypos can be accurate for up to one month $[7,18]$. Additionally, an unwillingness to admit to negative outcomes (i.e., social desirability bias) may have led some respondents to under-report hypos. For instance, a conscientious patient diagnosed with T2DM may view high rates of minor hypos as an indication that they are not adequately managing their illness. To reduce the likelihood of this, patients were informed that results were confidential and that their data would not be shared with their physician.

\section{CONCLUSIONS}

It is clear from the findings in the present, second wave of the GAPP2 survey that many patients with T2DM using basal insulin analogs, with or without bolus insulin, need additional advice and support to further reduce rates of self-treated hypoglycemia. The current consensus statement from a working group of the American Diabetes Association and the Endocrine Society describes a number of strategies that are known to help prevent hypoglycemia [4]. These include patient education, dietary intervention, exercise management, medication adjustment, glucose monitoring, and clinical surveillance. Each of these approaches is potentially valid for managing both minor and severe hypoglycemia. During consultations with health professionals, all patients should be proactively approached on the topic of hypoglycemia to help mitigate the risk of such events occurring. An important clinical consideration is also the regular measurement of markers of over-insulinization, such as weight gain.

Our findings therefore support the need for implementation of the strategies outlined in the consensus statement. Additional research is also required to further characterize the attributes of patients at risk for minor hypoglycemia, including personality, culture, glucose control, complexity of insulin regimen, and lifestyle factors. It will then remain to be determined how these factors may predict the psychological and behavioral responses observed in the current survey. 


\section{ACKNOWLEDGMENTS}

The GAPP2 survey was funded by Novo Nordisk, which supported the work of the authors, as well as the medical communications company (FTI Consulting) and research company (Bryter Research) that analyzed the data and provided medical writing and editing assistance in the preparation of the manuscript. Medical writing and editing services were also provided by Dr L Gillies and Gabrielle Parker of Watermeadow Medical (UK). All named authors meet the International Committee of Medical Journal Editors (ICMJE) criteria for authorship for this manuscript, take responsibility for the integrity of the work as a whole and have given final approval to the version to be published. All procedures followed were in accordance with the ethical standards of the responsible committee on human experimentation (institutional and national) and with the Helsinki Declaration of 1964, as revised in 2013. Informed consent was obtained from all patients included in the survey. The article processing charges for this publication were funded by Novo Nordisk.

Disclosures. Meryl Brod is a paid consultant to Novo Nordisk. Gagik Galstyan has received honoraria/consultation fees from Sanofi, Novo Nordisk, Lilly, Novartis, MSD, Astra Zeneca, Boehringer Ingelheim and Takeda and has served on advisory boards for MSD, Novo Nordisk and Sanofi. Ambika Gopalakrishnan Unnikrishnan has participated as a speaker in scientific sessions, clinical investigator and also as an advisor for Novo Nordisk as well as other pharmaceutical companies. Ilana Harman-Boehm has served on Novo Nordisk advisory boards and received honoraria for CME presentations from Novo Nordisk, Servier,
Sanofi-Aventis, Roche Pharmaceuticals, Eli Lilly, Novartis, Merck and Johnson and Johnson, along with lectures sponsored by Novo Nordisk. Vinay Prusty is employed by Novo Nordisk. Fernando Lavalle has participated as a formal advisor to Sanofi, Novo Nordisk, Janssen Cilag, Boehringer Ingelheim, MSD and Astra Zeneca; conducted research activities for Sanofi, Novo Nordisk, Janssen Cilag, Boehringer Ingelheim, MSD and Astra Zeneca and participated in speaker's bureaus for Sanofi, Novo Nordisk, Janssen, Pfizer, Astra Zeneca and Lilly. Margaret McGill has received honoraria for CME presentations for Novo Nordisk, Sanofi and MSD and has served on advisory boards for MSD and Abbott. Angela Murphy has served on Novo Nordisk advisory boards and received honoraria for CME presentations from Novo Nordisk, Servier, Sanofi-Aventis, Roche Pharmaceuticals, Eli Lilly, Novartis, Merck and Johnson and Johnson, along with lectures sponsored by Novo Nordisk. Felix Puchulu has participated in advisory boards for Novo Nordisk, Astra Zeneca, Sanofi and Janssen.

Compliance with Ethics Guidelines. All procedures conducted were in accordance with the ethical standards of the responsible committee on human experimentation (institutional and national) and with the Helsinki Declaration of 1964, as revised in 2013. Informed consent was obtained from all patients included in the survey.

Open Access. This article is distributed under the terms of the Creative Commons AttributionNonCommercial 4.0 International License (http://creativecommons.org/licenses/by-nc/4. $0 /$ ), which permits any noncommercial use, distribution, and reproduction in any medium, provided you give appropriate credit to the 
original author(s) and the source, provide a link to the Creative Commons license, and indicate if changes were made.

\section{REFERENCES}

1. Hayes RP, Fitzgerald JT, Jacober SJ. Primary care physician beliefs about insulin initiation in patients with type 2 diabetes. Int $\mathrm{J}$ Clinl Pract. 2008;62:860-8.

2. Karter AJ, Subramanian U, Saha C, Crosson JC, Parker MM, Swain BE, et al. Barriers to insulin initiation: the translating research into action for diabetes insulin starts project. Diabetes Care. 2010;33:733-5.

3. Brod M, Rana A, Barnett AH. Impact of self-treated hypoglycaemia in type 2 diabetes: a multinational survey in patients and physicians. Curr Med Res Opin. 2012;28:1947-58.

4. Seaquist ER, Anderson J, Childs B, Cryer P, Dagogo-Jack S, Fish L, et al. Hypoglycemia and diabetes: a report of a workgroup of the American Diabetes Association and the Endocrine Society. Diabetes Care. 2013;36:1384-95.

5. Brod M, Christensen T, Bushnell DM. Impact of nocturnal hypoglycemic events on diabetes management, sleep quality, and next-day function: results from a four-country survey. J Med Econ. 2012;15:77-86.

6. Brod M, Wolden M, Christensen T, Bushnell DM. A nine country study of the burden of non-severe nocturnal hypoglycaemic events on diabetes management and daily function. Diabetes Obes Metab. 2013;15:546-57.

7. Brod M, Christensen T, Thomsen TL, Bushnell DM. The impact of non-severe hypoglycemic events on work productivity and diabetes management. Value Health. 2011;14:665-71.

8. Willis WD, Diago-Cabezudo JI, Madec-Hily A, Aslam A. Medical resource use, disturbance of daily life and burden of hypoglycemia in insulin-treated patients with diabetes: results from a European online survey. Expert Rev Pharmacoecon Outcomes Res. 2013;13:123-30.

9. Jensen MM, Pedersen-Bjergaard U. Self-reported frequency and impact of non-severe hypoglycemic events in insulin-treated diabetic patients in Denmark. Diabetes Management. 2014;5:67-78.

10. Parekh WA, Ashley D, Chubb B, Gillies H, Evans M. Approach to assessing the economic impact of insulin-related hypoglycaemia using the novel local impact of hypoglycaemia tool. Diabet Med. 2015;32:1156-66.

11. Foos V, Varol N, Curtis BH, Boye KS, Grant D, Palmer JL, et al. Economic impact of severe and non-severe hypoglycemia in patients with Type 1 and Type 2 diabetes in the United States. J Med Econ. 2015;18:420-32.

12. Brod M, Peyrot M, Rana A, Barnett AH. GAPP2 ${ }^{\mathrm{TM}}$ : global survey of type 2 diabetes insulin analogue users shows extensive impact of self-treated hypoglycaemia and dosing irregularities on diabetes management in primary care. Prim Care Diabetes. 2013;7:78.

13. Munro N, Barnett AH. Incidence, worry and discussion about dosing irregularities and self-treated hypoglycaemia amongst HCPs and patients with type 2 diabetes: results from the UK cohort of the Global Attitudes of Patient and Physicians (GAPP2) survey. Int J Clin Pract. 2014;68:692-9.

14. Fulcher G, Singer J, Castaneda R, Fraige Filho F, Maffei L, Snyman J, et al. The psychosocial and financial impact of non-severe hypoglycemic events on people with diabetes: two international surveys. J Med Econ. 2014;17:751-61.

15. Levy AR, Christensen TL, Johnson JA. Utility values for symptomatic non-severe hypoglycaemia elicited from persons with and without diabetes in Canada and the United Kingdom. Health Qual Life Outcomes. 2008;6:73.

16. Leiter LA, Yale J, Chiasson J, Harris SB, Kleinstiver P, Sauriol L. Assessment of the impact of fear of hypoglycemic episodes on glycemic and hypoglycemia management. Can J Diabetes. 2005;29:186-92.

17. Sakane N, Kotani K, Tsuzaki K, Nishi M, Takahashi $\mathrm{K}$, Murata T, et al. Fear of hypoglycemia and its determinants in insulin-treated patients with type 2 diabetes mellitus. J Diabetes Investig. 2015;6: 567-70.

18. Brod M, Pohlman B, Wolden M, Christensen T. Non-severe nocturnal hypoglycaemic events: experience and impacts on patient functioning and well-being. Qual Life Res. 2013;22:997-1004. 